\title{
A walking support/evaluation machine for patients with parkinsonism
}

\author{
Yoshihiro Kai'), Tetsuya Tanioka'), Yoshio Inoue ${ }^{3)}$, Takuya Matsuda ${ }^{4)}$, \\ Kenichi Sugawara ${ }^{5)}$, Youichiro Takasaka ${ }^{6)}$, and Isao Nagamine ${ }^{2)}$ \\ ${ }^{1)}$ Department of Mechanical Engineering, Tokai University, Kanagawa, Japan ; Department of \\ Community \& Psychiatric Nursing,School of Medical Sciences, The University of Tokushima, \\ Tokushima, Japan; ${ }^{3}$ Department of Intelligent Mechanical Systems Engineering, Kochi University \\ of Technology; ${ }^{4)}$ Course of Intelligent Mechanical Systems Engineering, Department of Engineering, \\ Graduate School of Engineering, Kochi University of Technology, Kochi, Japan ; School of \\ Rehabilitation, Faculty of Health and Social Work, Kanagawa University of Human Services, \\ Kanagawa, Japan ; and ${ }^{6}$ Psychiatry and Neurology Hosogi Unity Hospital, Kochi, Japan
}

\begin{abstract}
Various walk supporting systems have been devised and developed. However, they have not been designed for supporting or evaluating the gait of parkinsonian patients, and not much consideration has been given to gait disturbances of parkinsonian patients. In this study : (a) We prepared a tentative model of walk supporting and monitoring system in consideration of typical symptoms of parkinsonism. (b) We conducted gait rehabilitation in a parkinsonian patient using the walk supporting and monitoring system and confirmed (i) the occurrence of frozen gait during walking, (ii) brachybasia, (iii) the absence of anterior tilting of the posture, pulsion symptom, and festination, and (iv) occurrence of hesitation to start walking. Therefore, typical symptoms of parkinsonism can be detected by the use of this system. (c) The medical staff can evaluate the state of recovery of patients on the basis of the data obtained from this system and use them for purposes such as guidance of rehabilitation. J. Med. Invest. 51 : 117-124, February, 2004
\end{abstract}

Keywords : sensor-controlled walker, walking support/evaluation machine, parkinsonism

\section{INTRODUCTION}

Patients with parkinsonism having gait disturbances are likely to fall, because they walk in an anteriorly tilted posture in quick short steps, and show typical symptoms such as "festination", in which they walk faster and faster regardless of their will, and "frozen gait", in which they suddenly become unable to move the feet forward as if they were stuck to the floor (1). Therefore, physiotherapists and nurses (medical staff)

Received for publication October 7, 2003 ; accepted December 5, 2003.

Address correspondence and reprint requests to Yoshihiro Kai, Department of Mechanical Engineering, Tokai University, Hiratsukashi, Kanagawa 259-1292, Japan and Fax : +81-463-59-2207. make such great efforts in gait rehabilitation of parkinsonian patients that the care of parkinsonian patients turns out to be their major burden. Also, as the elderly population of the society increases further, and as patients who need gait rehabilitation increase, the burden of their care on the medical staff is expected to increase, and the time that can be allotted to gait rehabilitation of each patient may become insufficient.

Therefore, to promote gait rehabilitation of parkinsonian patients with gait disturbances and to reduce the burden of their gait rehabilitation on the medical staff, the development of walk supporting systems that allow patients to train themselves in walking is desired. Also, as collecting reference data for objective evaluation of the state of recovery of patients and providing 
them to the medical staff in addition to simply supporting patients in their gait rehabilitation is considered to be very advantageous for guidance of gait rehabilitation, systems that perform such tasks are also anticipated.

Various walk supporting systems have been devised and developed (2-4). However, they have not been designed for supporting or evaluating the gait of parkinsonian patients, and not much consideration has been given to gait disturbances of parkinsonian patients.

In this study, we first evaluated typical gait disturbances of parkinsonian patients and a walk supporting and monitoring system suited for such gait disturbances. Next, we presented a model of walk supporting and monitoring system that we tentatively prepared in consideration of these gait disturbances. We further applied this system to 5 healthy subjects and 1 patient with parkinsonism and evaluated the effectiveness of the system on the basis of the results obtained. A patient who could freely move the upper limbs were selected as the subject.

\section{CHARACTERISTICS OFGATT DISTURBANCES IN PARKINSONIAN PATIENTS}

Parkinsonism (1) is a general name of a group of diseases that affect the control of the motor system and present with the 4 major symptoms of (a) resting tremor (tremor of hands and feet in a stationary state), (b) muscle rigidity, (c) bradykinesia or akinesia, and (d) disorders of maintaining the posture. It is a concept that includes idiopathic parkinsonism, or a disease that presents with the above motor disorders as characteristic symptoms due to impairment of the central nervous system, particularly dopaminergic neurons, and symptomatic parkinsonism, or a condition that presents with symptoms very similar to those of idiopathic parkinsonism due to cerebrovascular disorders or the administration of drugs (e.g. some antipsychotics, drugs of the digestive system, and depressors). The number of patients with parkinsonism is estimated to be 10 million in the world, and with increases in the elderly population, more than 40 million people are predicted to develop this disease by the year 2020 (6).

Parkinsonism is treated primarily by drug therapy. However, drug therapy is associated with the problem of adverse reactions, and simultaneous physiotherapy (including gait rehabilitation) to complement the drug therapy is important. Major characteristics of gait disturbances in parkinsonian patients are an an- teriorly tilted posture with little arm swinging, short strides, quick short steps, and reduced lifting of the toes from the ground, which all make them prone to stumbling (1). Typical symptoms often noted clinically include frozen gait, hesitation to start walking, festination, and pulsion symptoms (7-9).

Frozen gait is the phenomenon in which the patient suddenly becomes unable to bring the feet forward and tremble them in their positions. As the patient tries harder to continue walking, it becomes more difficult to lift the feet, the upper part of the body if further tilted forward, the feet only make small steps in the same position, and the patient, being unable to step forward, may eventually fall. Frozen gait may be observed at the beginning of and during walking, but frozen gait at the beginning of walking is called hesitation to start walking. Festination is the phenomenon in which the patients involuntarily accelerate as they continue to walk and seems as if they started running. Pulsion symptom is the phenomenon in which the patients tilt forward until they start rushing, being unable to maintain the position. Since the patients rush while hardly lifting the toes, they are likely to fall.

Therefore, patients with parkinsonism are considered likely to fall (a) due to stumbling or (b) due to frozen gait, or inability to step forward, as in Fig.1. Therefore, the walk supporting and monitoring system must first prevent falling such as is shown in Fig.1. Also, it must prevent festination and pulsion symptom. Moreover, if aspects of walking such as the occurrence of frozen gait can be monitored, the state at the onset

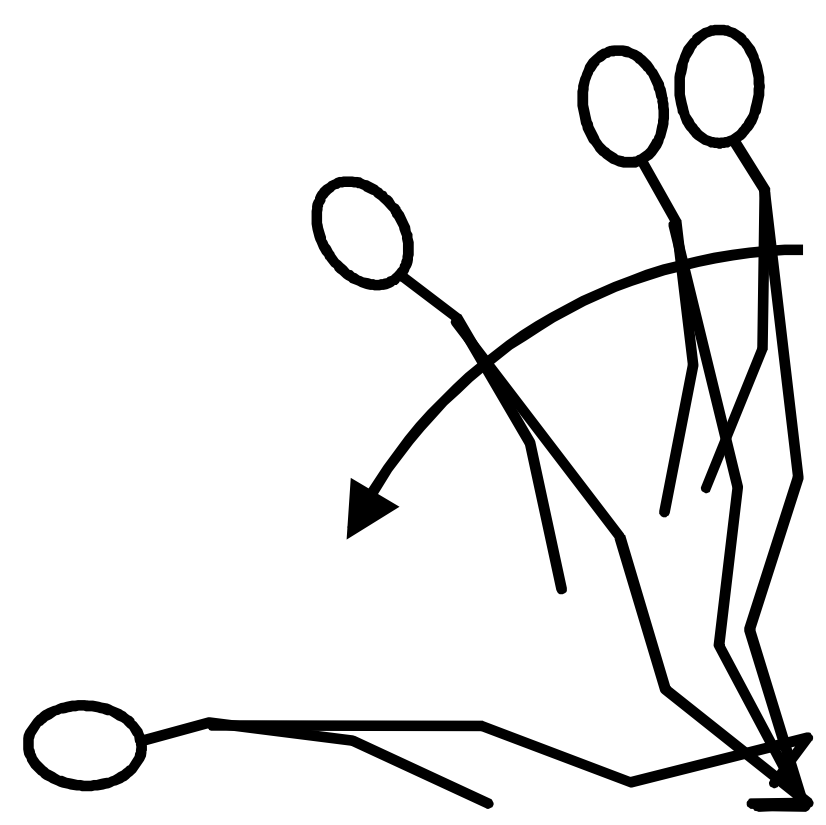

Fig. 1. A parkinsonian's fall 
of gait rehabilitation (or medication) can be compared with the state after 1 week or 1 month. These data are considered to be useful for the medical staff in evaluation of the effectiveness of drug therapy or gait rehabilitation.

\section{WALK SUPPORTING AND MONITORING SYSTEM}

In this study, in consideration of the above gait disturbances of parkinsonian patients, we prepared a tentative model of walk supporting and monitoring system. Fig.2 shows the appearance of this walk supporting and monitoring system. This system was prepared by attaching motors on both rear wheels and a motion controller at the front part of a commercial walker (JHS-1, Hitachi Chemical Co., Ltd.). Its two front wheels were free casters. As safety measures, it was equipped with 4 emergency stop buttons that could be pushed by care-givers and a brake that acted when gripped by the patient. In addition, force sensors,
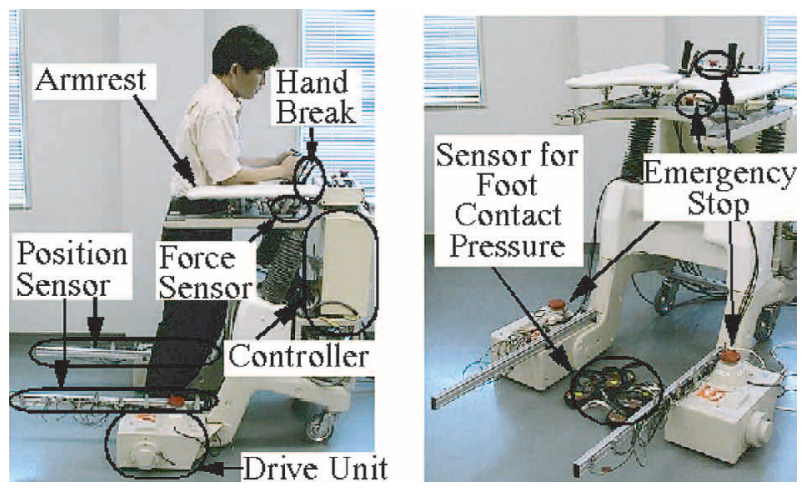

Fig. 2. A walk supporting and monitoring system

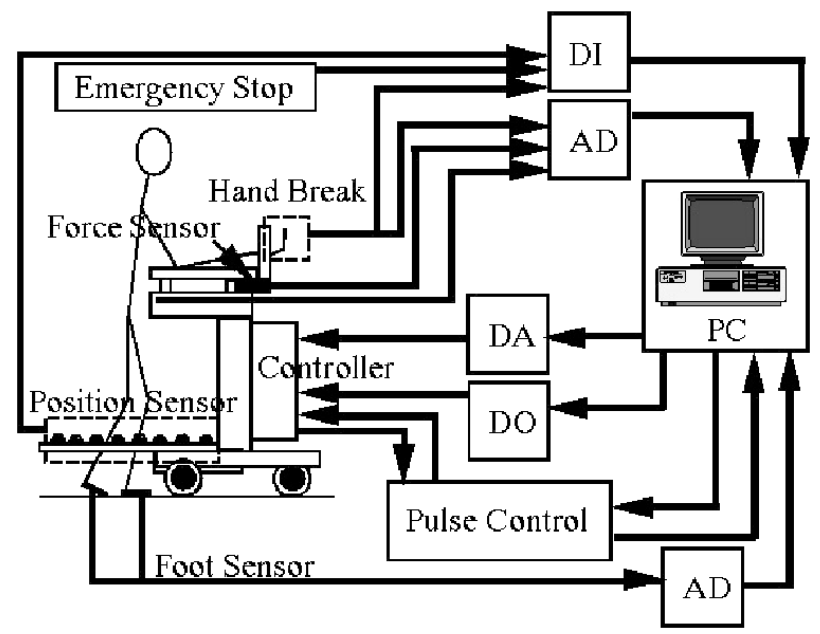

Fig. 3. Control mechanisms of walk supporting and monitoring system foot contact pressure sensors, and position sensors described below were also attached to allow real-time control of the walk supporting and monitoring system based on the information from the sensors. Fig.3 shows the control mechanisms incorporated in the walk supporting and monitoring system.

The measures for gait disturbances in parkinsonian patients installed in this system are shown below.

\section{1)Measures against the anterior tilting of the posture}

Parkinsonian patients are likely to take an anteriorly tilted posture and need some support to prevent falling forward. Therefore, a walker with armrests was used as the basis of this walk supporting and monitoring system, and anterior tilting of the posture was prevented by adjusting the height of the armrests according to the height of the patient.

\section{2)Detection of the patient's will to move}

The walk supporting and monitoring system is required to detect the patient's will to "walk" and to operate according to it. In this study, force sensors

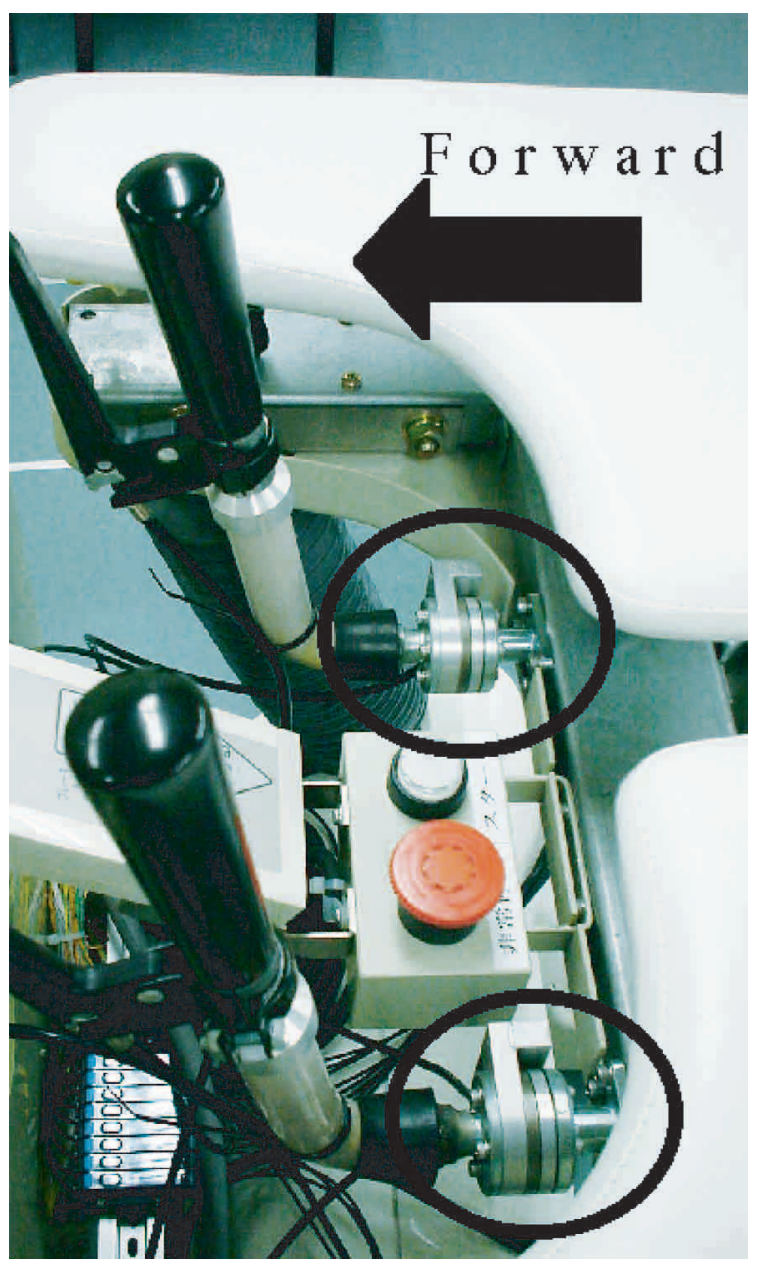

Fig. 4. Force sensors 
(Mini 8/40, BLAutotech, Inc.) were used to detect the patient's will to move. A force sensor was attached to the base of each of the left and right handlebars (Fig.4) and collected the information about the force with which the patient pushed or pulled the handlebars.

The walk supporting and monitoring system was driven according to this force information. More specifically, when the combined force applied to the left and right handlebars $(\mathrm{Fp})$ to the anterior direction exceeds a predetermined level $(\mathrm{Fa})$,

$$
\mathrm{Fp}>\mathrm{Fa}
$$

the walk supporting and monitoring system was driven forward, and when $\mathrm{Fp}$ was less than $\mathrm{Fb}$,

$$
\mathrm{Fp}<\mathrm{Fb}
$$

the walk supporting and monitoring system was driven backward. This mechanism is considered to allow the patient to drive the walk supporting and monitoring system intuitively by applying force to the handlebars without changing the posture during rehabilitation. However, when the medical staff considered it dangerous to drive the system driven backwards, the system could be adjusted not to move backwards.

\section{3)Measures against festination and pulsion symptom}

The walk supporting and monitoring system was designed to be driven at a fixed speed so that it would serve as a pacemaker and prevent festination and pulsion symptom. If a condition represented by the above equation (a) or (b) was fulfilled, the system was driven at a fixed speed.

\section{4)Prevention of falling and detection of anterior tilting of the posture and brachybasia}

When parkinsonian patients use a conventional walker, they are likely to fall as they cannot step forward due to "stumbling" or "frozen gait" and the feet are left behind the walker as shown in Fig.5. Therefore, we attached position sensors to detect the relative distance between the patient's feet and the system and to stop the system and prevent the patient from falling

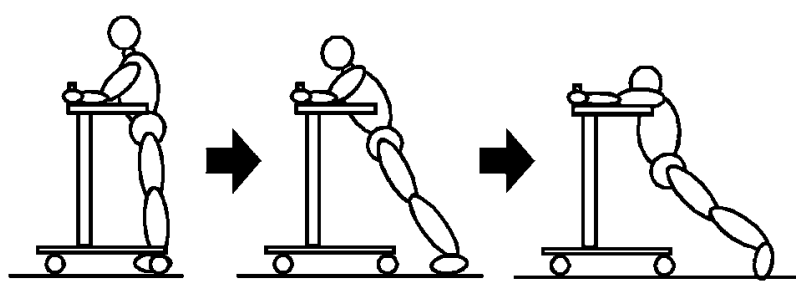

Fig. 5. A fall of parkinsonian patient when using conventional walker

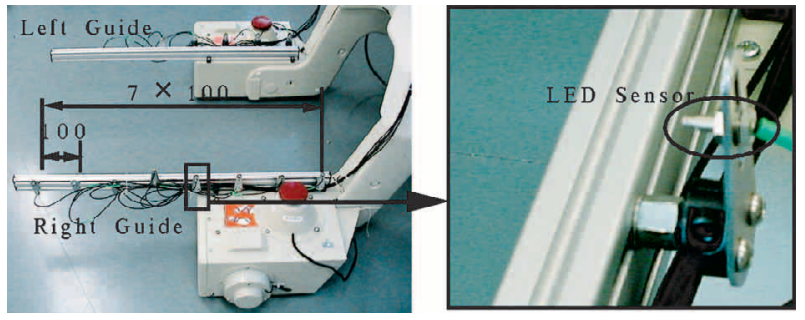

Fig. 6. LED position sensors

when the patient's feet were left behind the system (Fig.6).

We attached 8 light emitting diode (LED) sensors (FS-V 11, FS-V 12, Keyence Co.) at equal intervals $(100 \mathrm{~mm})$ on the bilateral guides placed in the lower part of the system. LEDs were aligned on the left guide, and light receivers on the right guide, and the positions of the patient's feet are detected as they blocked the light between the light emitters and receivers.

Falling was prevented as follows. The $n$th sensor from the body of the system was determined as the fall-prevention position, and the system was stopped when the $n$th or a farther rear sensor detected the patient's foot (Fig.7).

In addition, as the patient must hold the handlebars of the walk supporting and monitoring system during walking, the patient's feet shift to the rear of the system if the patient walks in an anteriorly tilted posture. Thus, the patient is considered to be walking in an anteriorly tilted posture if only rear sensors respond (Fig.8 (a)).

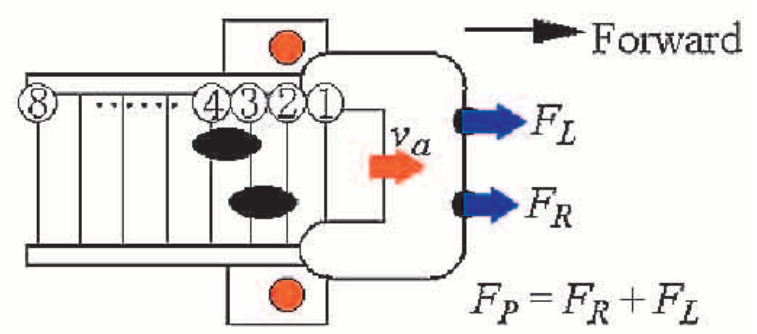

$F_{P}>F_{a}:$ Forward, $F_{P}<F_{b}:$ Backward $v_{a}:$ Constant Speed

(a) Move

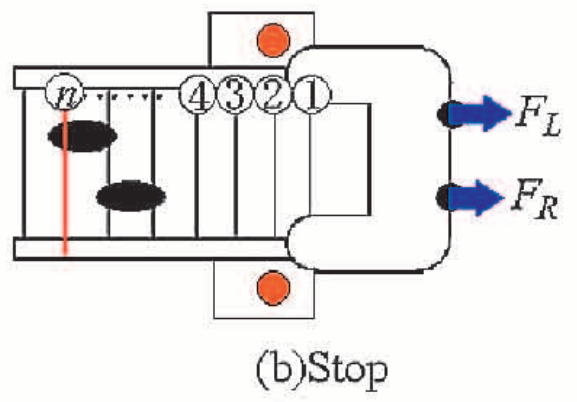

Fig. 7. A fall prevention control system 
Also, if the patient walks with small steps, only a small number of sensors respond, so that brachybasia can also be detected (Fig.8(b)).

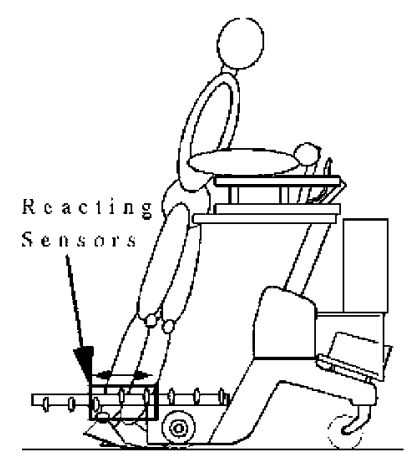

(a) Anterior Tilted Posture

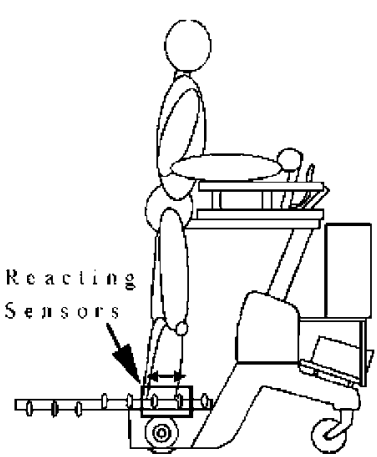

(b) Brachybasia
Fig. 8. A detection system for anterior tilted posture and brachybasia

\section{5)Detection of frozen gait and hesitation to start walking}

In the gait of normal people, heel contact, foot contact, heel off, and toe off are repeated in nearly constant gait cycles (Fig.9) (10). However, when a parkinsonian patient shows frozen gait or hesitation to start walking, the patient suddenly becomes unable to move the feet forward and trembles the feet at the same position, so that regular landing and take-off cycles such as those of healthy individuals disappear.

Therefore, in this study, we aimed to detect frozen gait or hesitation to start walking according to pressure information on the planta and made the plantar pressure

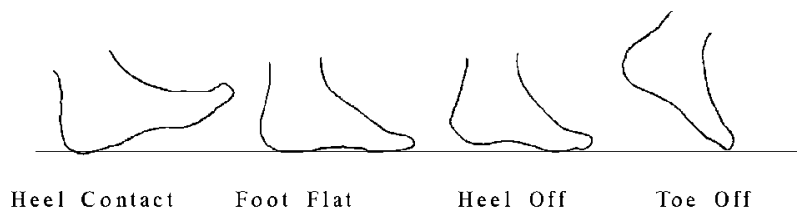

Fig. 9. Gait cycle of normal people
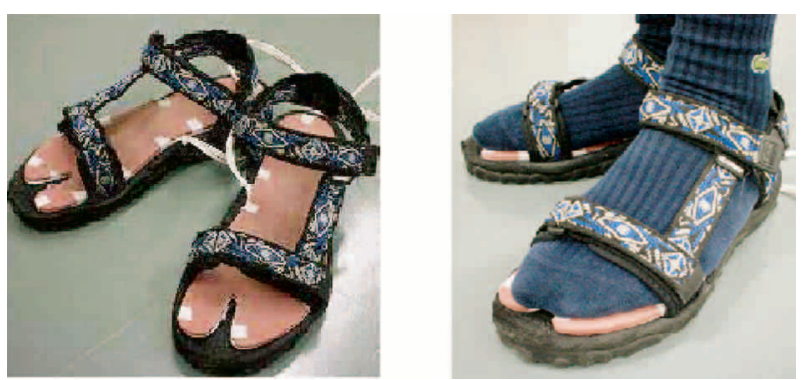

Fig. 10. Heel-to-toe pressure sensors sensor pads (Heel-to-toe pressure sensors) shown in Fig.10. The information obtained through the sensors incorporated in the pads was transmitted to the computer of the walk supporting and monitoring system via an $\mathrm{AD}$ conversion board.

Six small pressure sensors (PS-10KAM183, Kyowa Electric Industries) were embedded between the sponge insole and the plastic board, and this sensor pad was fixed on a sandal with an adhesive (Fig.11). To minimize the limitation due to the individual variation in the size or the shape of the foot, sandals with adjustable straps were used. The pressure sensors were placed at the heel, Chopart's joint, first metatarso-phalangeal (MP) joint, 4 th MP joint, big toe, and third toe on the basis of the shift of the plantar pressure reported by Turu et al. (11) and Nagata et al. (11) Fig.12 shows the positions of the sensors.

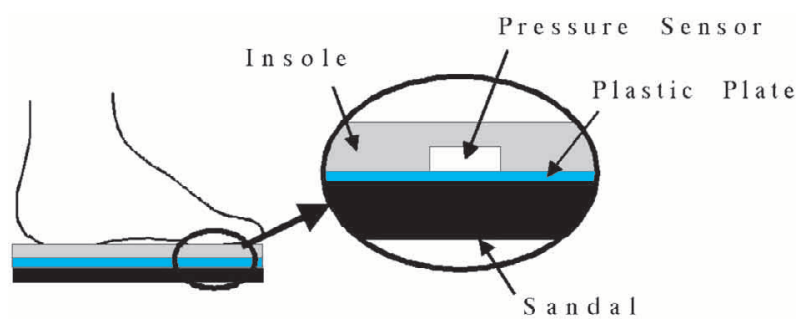

Fig. 11. Structure of heel-to-toe pressure sensors

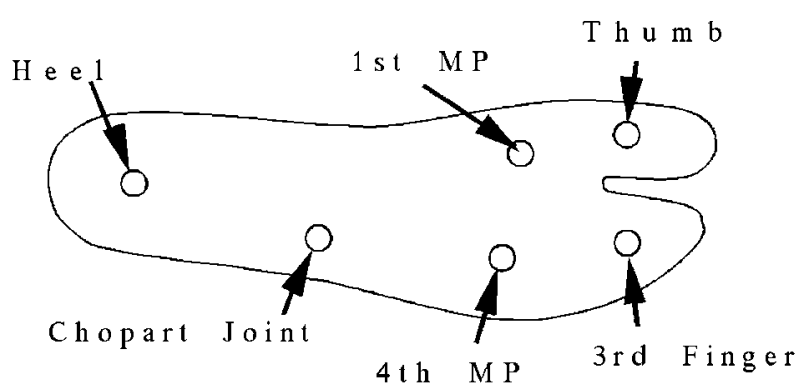

Fig. 12. Positions of sensors

\section{EXPERIMENTS}

\section{1)Experimental procedures}

Five healthy individuals (males aged 21-39 years) and 1 patient with parkinsonism walked with the walk supporting and monitoring system, and the effectiveness of the system was evaluated according to the results obtained. In the 5 healthy subjects, the mean age was 26.2 years (standard deviation 7.23 years), mean height was $165.4 \mathrm{~cm}$ (standard deviation 3.91 $\mathrm{cm}$ ), and the mean body weight was $53 \mathrm{~kg}$ (standard deviation $4.90 \mathrm{~kg}$ ). The patient was a 76-year-old male who was $165 \mathrm{~cm}$ tall and weighed $53 \mathrm{~kg}$. He had mild muscle rigidity, akinesia, and frozen gait, and the se- 
verity of the disease was stage 3 according to the severity scale of Hoehn and Yahr (13).

In this study, gait of the patient was evaluated by a physician and a physiotherapist, and the speed of the walk supporting and monitoring system was determined at $0.20 \mathrm{~m} / \mathrm{s}(\approx 0.72 \mathrm{~km} / \mathrm{h})$ in consideration of the mean maximum walking speed of individuals of the patient's age (14). The fall prevention position $(n)$ was set at 8 . The sampling time was $0.01 \mathrm{sec}$. Each subject was instructed to be relaxed during test walking.

The height of the armrests was adjusted before the test so that the subject would not tilt forward. Each of the plantar pressure sensors was confirmed to be near the intended site of the subject's planta. During the test, 2 physiotherapists accompanied the patient on both sides to avoid danger. A physician and a nurse were also present at the scene of the test. Careful informed consent was obtained from the patient before the execution of the test.

\section{2)Results}

In the parkinsonian patient, (a) frozen gait during walking was confirmed, (b) brachybasia was confirmed, (c) anterior tilting of the posture, pulsion symptom, or festination was not observed, and (d) hesitation to start walking was confirmed.

In the 5 healthy subjects, the stride varied among individuals, but little difference was observed in other
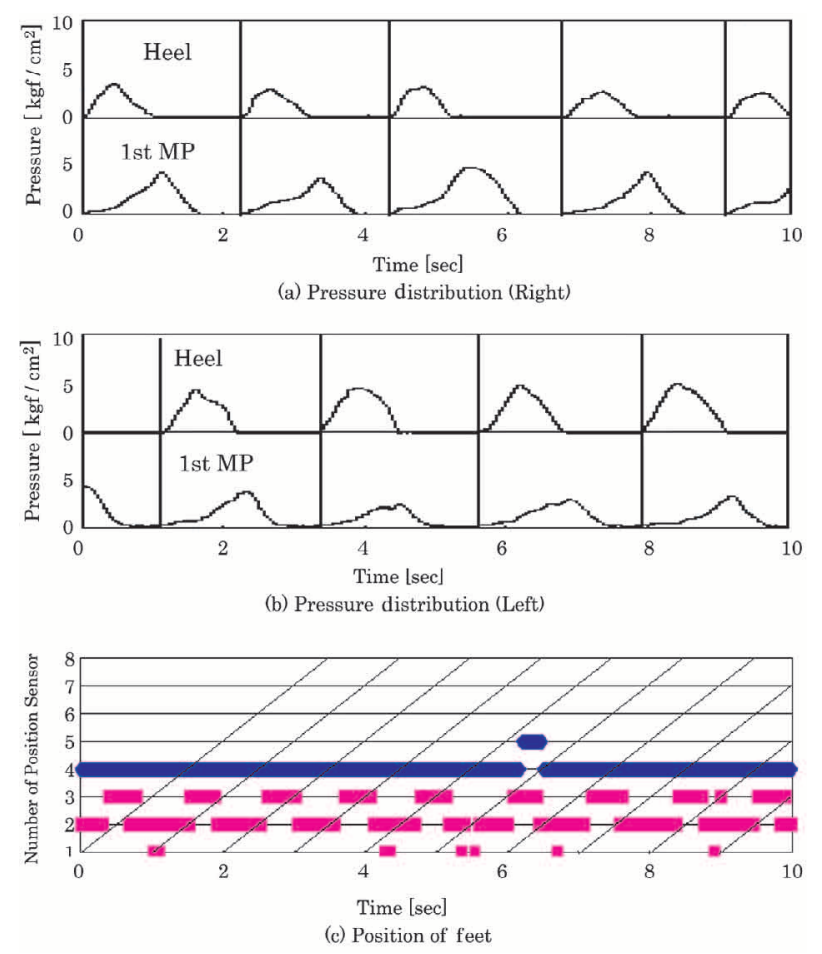

Fig. 13. Results in a healthy subject

"The straight lines, which have the slope of $20[\mathrm{~cm} / \mathrm{sec}]$, show the trajectories of objects at rest seen from the machine."
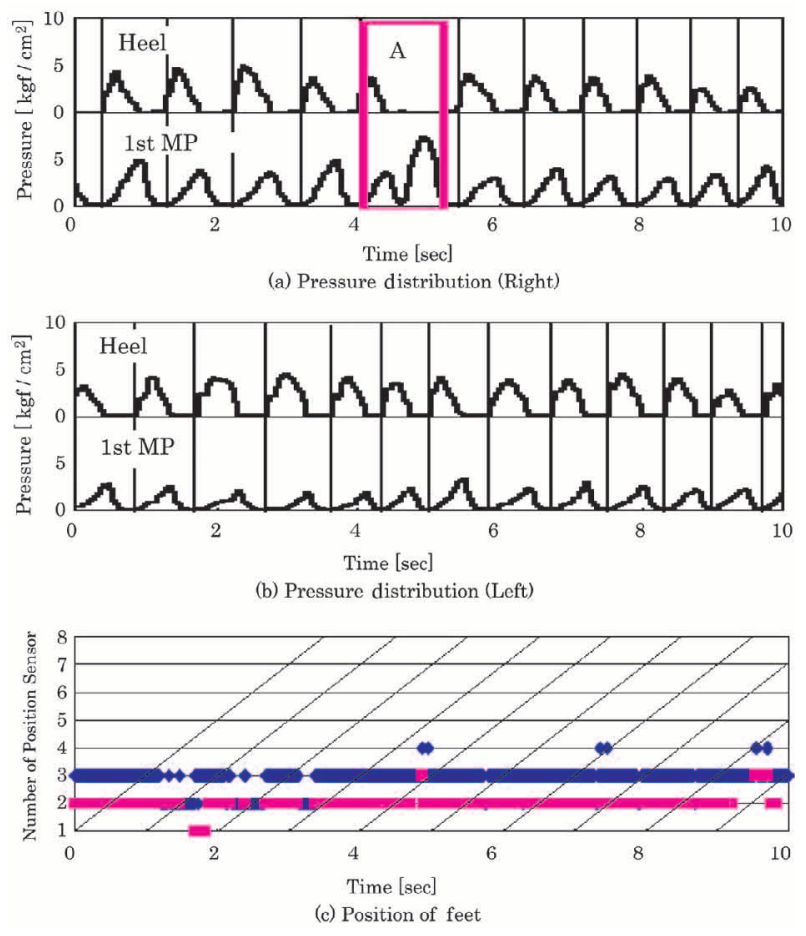

Fig. 14. Results in a parkinsonian patient

"The straight lines, which have the slope of $20[\mathrm{~cm} / \mathrm{sec}]$, show the trajectories of objects at rest seen from the machine."

aspects of the gait. Fig.13 shows the results of measurements during walking in subject $A$ (age 24 years, height $163 \mathrm{~cm}$, weight $46 \mathrm{~kg}$ ), who walked with the shortest steps among the 5 healthy subjects. Fig. 14 shows the results in the parkinsonian patient. In Figs.13 and 14, the horizontal axis is the scale of time; (a) shows the pressures at the heel and first MP joint of the right foot, and (b) shows those of the left foot. Although pressures were measured at 6 sites on the planta of each foot in this study, the figures show the pressures only at the heel and first MP joint, where frozen gait was observed most conspicuously in the parkinsonian patient. In each figure, (c) shows the position of the feet. The sensors, numbered serially from the most forward to the most rear ones, are shown along the vertical axis. Also, in (c) of each figure, the positions of the anterior and posterior feet are shown with $\square$ and $\checkmark$, respectively. For example, if the feet of a subject were positioned at time $\mathrm{t}$ as in Fig. 8 (a), the $2 \mathrm{nd}, 3 \mathrm{rd}$, and 4 th sensors responded, and line 2 (number of the most anterior sensor that responded) is marked with at time $t$, and line 4 (number of the most posterior sensor that responded) is marked with $\diamond$.

Fig. 15 shows the results of measurements of the plantar pressure that indicate hesitation to start walking in the parkinsonian patient. 


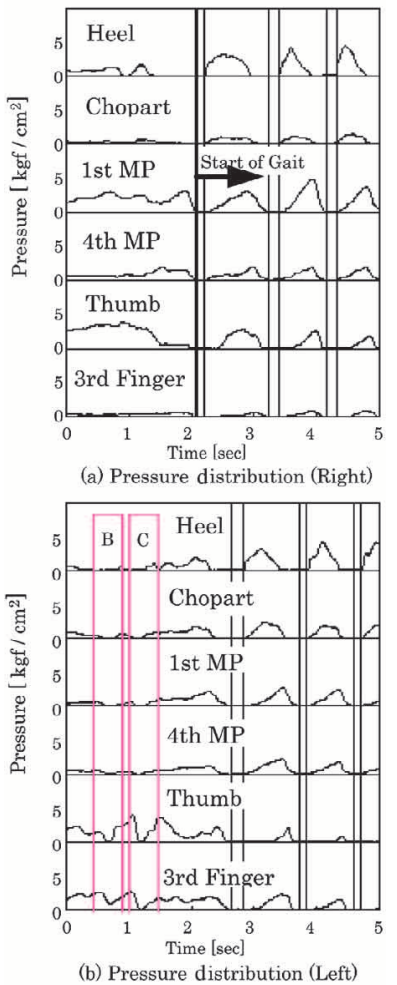
Fig. 15. Hesitation to start
walking

\section{DISCUSSION}

\section{1)Frozen gait during walking}

The parkinsonian patient showed frozen gait during walking. This could be detected in the graph of plantar pressures as follows. First, in the healthy subject A (Fig.13(a), (b)), heel contact, an increase in the pressure at the heel, a decrease in the pressure at the heel with an increase in the pressure at the MP joint, and a decrease in the pressure at the MP joint were repeated in nearly identical gait cycles. As mentioned above, this is a normal gait pattern. In the parkinsonian patient, on the other hand, the pressure at the MP joint of the right foot decreased but increased again immediately before it became zero (the MP joint is lifted) in the right foot in segment A of Fig.14(a). Also, while an increase in the pressure at the MP joint was observed after an increase in the pressure at the heel, no increase in the pressure at the heel was observed in Segment A. Thus, as shown in Fig.16, the patient tried to lift the toes after lifting the heel, but could not lift the toes, and stepped on the MP region again at

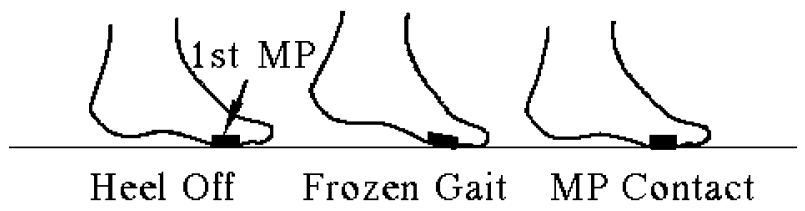

Fig. 16. Frozen gait the same position (frozen gait).

\section{2)Brachybasia}

When Fig.13(c) and Fig.14(c) are compared, the healthy subject $A$ is known to have moved the feet evenly over the $2 \mathrm{nd}, 3 \mathrm{rd}$, and 4 th sensors. In contrast, the feet of the parkinsonian patient were often located at the 2nd and 3rd sensors, suggesting that the patient was walking with shorter steps than subject A. Thus, the information obtained from the position sensors permits judgment of whether the subject has brachybasia or not.

The shortening of the stride of the parkinsonian patient could also be confirmed from the data of the plantar pressure. Figs.13 and 14 show data of the healthy subject $A$ and the patient during a 10 second period. Since the speed of the walk supporting and monitoring system was fixed at $0.2 \mathrm{~m} / \mathrm{s}$ in both subjects, the system moved $2 \mathrm{~m}$ during the 10 seconds in both cases. The healthy subject walked this distance in about 9 steps (Fig.13(a),(b)), but the patient walked the same distance in almost 24 steps (Fig.14(a),(b)). The patient needed more than twice the number of steps to walk the same distance. Thus, the data of the plantar pressure also confirmed that the parkinsonian patient walked with shorter steps than the healthy subject.

\section{3)Anterior tilting, pulsion symptom, and festination}

As mentioned in Walk supporting and monitoring system, Section 4 , the feet of the subject are considered to be located near the back of the walk supporting and monitoring system when the patient walks in an anteriorly tilted position. In this study, however, the patient's feet were located at the 2 nd and 3 rd sensors in the anterior part of the system. Therefore, the patient is considered not to have tilted forward during walking. When a subject tilts forward, he tends to tiptoe and does not apply sufficient pressures to the heels. The patient in this study applied sufficient pressures at the heels except in Segment A of Fig.14 (a) (frozen gait), and the data of the plantar pressure also disagreed with anterior tilting of the patient. Also, because of the absence of anterior tilting of the posture, pulsion symptom as a result of anterior tilting was not observed. Moreover, if festination occurs, the speed of walking increases progressively, the patient appears as if he started running, and the duration of the gait cycle (time from a heel contact to the heel contact of the next step) becomes shorter and shorter. However, festination is considered not to have occurred in our patient, because the gait cycle remained nearly constant except in segment A of Fig.14 (a), (b) (frozen gait). 


\section{4)Hesitation to start walking}

In Fig.15, the pressures at the heel, Chopart's joint, 1 st MP joint, and 4 th MP joint of the left foot were nearly zero in segments $\mathrm{B}$ and $\mathrm{C}$, but the pressures at the big toe and 3 rd toe increased again after a decrease. These results suggest that the patient lifted the heel, Chopart's joint, 1 st MP joint, and 4 th MP joint and tried also to lift the big toe and $3 \mathrm{rd}$ toe but could not and stepped again on the big toe and $3 \mathrm{rd}$ toe. The patient then supported himself on the left foot and started walking by swinging the right foot. Thus, evaluation of the data of the plantar pressure before the start of walking allows the judgment of whether the patient had hesitation to start walking or not.

Changes in gait such as a decrease in the frequency of the occurrence of frozen gait or hesitation to start walking or widening of the stride can be confirmed by comparing the data obtained from the walk supporting and monitoring system immediately after the beginning of rehabilitation (or medication) with those obtained after 1 week or 1 month. Therefore, the data obtained from this system are expected to be useful for evaluation of the effectiveness of rehabilitation and medication by the medical staff.

\section{CONCLUSIONS}

In this study :

(1) We prepared a tentative model of walk supporting and monitoring system in consideration of typical symptoms of parkinsonism.

(2) We conducted gait rehabilitation in a parkinsonian patient using the walk supporting and monitoring system and confirmed (i) the occurrence of frozen gait during walking, (ii) brachybasia, (iii) the absence of anterior tilting of the posture, pulsion symptom, and festination, and (iv) occurrence of hesitation to start walking. Therefore, typical symptoms of parkinsonism can be detected by the use of this system.

(3) The medical staff can evaluate the state of recovery of patients on the basis of the data obtained from this system and use them for purposes such as guidance of rehabilitation.

\section{REFERENCES}

1. Mano, Y: Falling of the elderly and preventive measures, Ishiyaku Shuppan, Tokyo, 1999, pp.146152 (in Japanese)
2. Tani T, Sakai A, Koseki A, Hattori S, Fujie M : Evaluation of walking tracks for training using active impedance, Kiron (version C), 63(613) (in Japanese) : $3168-3173,1997$

3. Nemoto Y, Egawa S, Koseki A, Hattori S, Ishii T, Fujie M: Power-assisted Walking Support System for Elderly, Proc. 20 th Int. Conf. IEEE EMBS, 20(5) : 2693-2695, 1998

4. Miyawaki K, Iwami T, Obinata G, Kondo Y, Kutsuzawa K, Ogasawara Y, Nishimura S : Evaluation of the Gait of Elderly People Using an Assisting Cart (Gait on Flat Surface), JSME Int J Ser C 43(4) : 966-974, 2000.

5. Association of Clinical Gait Analysis, ed.: Introduction to Rehabilitation Programs for Gait-Related Disorders, Ishiyaku Shuppan, Tokyo, 1999, pp.212215 (in Japanese)

6. Morris ME, Movement Disorders in People with Parkinson Disease : A Model for Physical Therapy, Physical Therapy 80 (6) :578-597, 2000

7. Ueno E: Gait disturbances in Parkinson's disease: Brachybasia, frozen gait, gait apraxia, Noshinkei 43 (8) (in Japanese) : 720-729, 1991

8. Ueno $\mathrm{E}$ : Clinical care and preventive measures against gait disturbances in the elderly : Brachybasia and frozen gait. Medical Practice 12 (3) (in Japanese) : 357-360, 1995

9. Nakanishi R, Mitsuishi T, Yamanaga H, Ideta $\mathrm{T}$ : Neuromuscular diseases and dysbasia, Parkinson's disease, motor ataxia, and myotonic dystrophy. Journal of Clinical Rehabilitation 5 (7) (in Japanese) : 678-683, 1996

10. Japan Society of Prosthetics (ed) : Biomechanics of prostheses of the lower limbs : Basic dynamics of the gait of hemiplegic patients and prostheses, Ishiyaku Shuppan, Tokyo, 1996, pp.2-4 (in Japanese)

11. Turu T, Yamakuma K, Oniki Y : Dynamic Pressure Distribution beneath the Foot during Walking. Orthopedic and Traumatology 39 (2) : 839842,1990

12. Nagata A: Biokinetics : From exercise dynamics to rehabilitation technology. Kyorin Shoin, Tokyo, 1996, pp.103-124 (in Japanese)

13. Hoehn MM, Yahr MD : Parkinsonism : onset, progression and mortality. Neurology 17 : 427442, 1967

14. Nakamura R, Saito $\mathrm{H}$ : Clinical Kinesiology, Second Edition, Ishiyaku Shuppan, Tokyo, 2000, pp.220-222 (in Japanese) 